\title{
Können wir die Diabetes-Welle stoppen?
}

\section{Ein Gespräch mit dem Ernährungsmediziner Dr. Matthias Riedl}

\section{Bei Kohlenhydratstoffwechselstörun- gen sollten Kohlenhydrate reduziert und durch gesunde ersetzt werden - Bereits mit kleinen Veränderungen lassen sich große Effekte erzielen.}

\section{Sie haben sich als Internist auf die Diabetologie und die Ernährungs- medizin spezialisiert. Was hat Sie speziell an der Diabetologie gereizt?}

Die Diabetologie war vor 30 Jahren, als ich anfing, ein absolutes Mangelgebiet. Die Versorgung der Menschen mit Diabetes war damals ähnlich schlecht wie für die ernährungsbedingten Erkrankungen heute. Die Behandlungsergebnisse führten zu suboptimalen Ergebnissen - aus Mangel an Betreuung. Deshalb habe ich mich dafür entschieden.

\section{Diabetes Typ 2 hat in den letzten Jahrzehnten stetig zugenommen. Und es erkranken immer mehr jüngere Menschen. Gibt es Daten dazu, in welchen Größenordnungen dies der Fall ist?}

Ja. Es liegen z. B. Daten aus einer US-amerikanischen Untersuchung bei Kindern und Jugendlichen vor: Bei den Übergewichtigen hat man festgestellt, dass $4 \%$ bereits an Diabetes erkrankt waren und $25 \%$ eine Störung im Glukosestoffwechsel aufwiesen. Das bedeutet, rund ein Drittel der übergewichtigen Jugendlichen hat Störungen im Glukosestoffwechsel, eine gestörte Glukosetoleranz oder bereits Diabetes.

In Deutschland liegt die Erkrankungsrate übergewichtiger Kinder und Jugendlicher etwas niedriger, bei ca. 2\%. Aber 10-20\% unserer Kinder und Jugendlichen sind übergewichtig, das ist sehr bedenklich.

\section{Was sind die entscheidenden Ursachen, dass die Menschen immer mehr zunehmen?}

Es ist das Missverhältnis von diabetogen und antidiabetogen wirkenden Nahrungsmitteln. Wir haben es mit einer Kohlenhydratstoffwechselstörung zu tun. Das hat die Diabetologie erstaunlicherweise sehr lange ignoriert. Es scheint banal, aber eine Koh- lenhydratstoffwechselstörung sollten wir behandeln, indem wir die Kohlenhydrate reduzieren.

\section{Warum wurde das so lange ignoriert?} Als ich begann, in der Diabetologie zu arbeiten, lautete die Empfehlung führender Diabetologen in Deutschland: So viel Lebensqualität wie möglich. Gleichzeitig ging der zunehmende Wohlstand in dieser Zeit mit einem zunehmenden Kohlenhydratverzehr einher. Das hat dazu geführt, dass bei vieIen Patienten hohe Insulindosen notwendig wurden. Ein nicht unerheblicher Teil hat unter der hohen Insulindosierung zugenommen.

Es herrschte die Ansicht vor: Diabetes heißt zwar Zuckerkrankheit, werde aber nicht durch den Verzehr von Kohlenhydraten verursacht. Aber Kohlenhydrate werden auch zu Zucker verstoffwechselt, besonders die prozessierten Kohlenhydrate, die massenhaft verzehrt werden.

Wenn Sie es konkret machen, wie sollte die Ernährung bei einer Kohlenhydratstoffwechselstörung aussehen?

Bei einer Kohlenhydratstoffwechselstörung sollten die Kohlenhydrate reduziert und durch gesunde ersetzt werden. Kohlenhydrate in Gemüse, Nüssen oder Pilzen wirken von Natur aus antidiabetogen. Der Schlüssel ist eine artgerechte Ernährung. Wenn wir uns anschauen, was Naturvölker heute verzehren, sind wir bei der Definition: bis zu 500 Gramm Gemüse, zuckerarme Beeren, Nüsse und Pilze; die richtige Eiweißmenge $(0,8-1,2 \mathrm{~g} / \mathrm{kgKG})$ aus überwiegend pflanzlichen Eiweißquellen. Das sind die Schieberegler. Regeln wir das Gemüse hoch, haben wir automatisch weniger Übergewicht und weniger Zivilisationskrankheiten. Regeln wir das pflanzliche Eiweiß hoch, haben gerade Diabetiker ein besseres kardiovaskuläres Outcome. Regeln wir aber den Konsum von rotem Fleisch hoch, steigt das Risiko für Diabetes, Arteriosklerose und Krebs. Pflanzliche Öle und Fette sind freigegeben, sie sind mit weniger Krebs und weniger Arterienverkalkung assoziiert, und Fette aus Nüssen sogar mit weniger Gewicht. Für Zu-

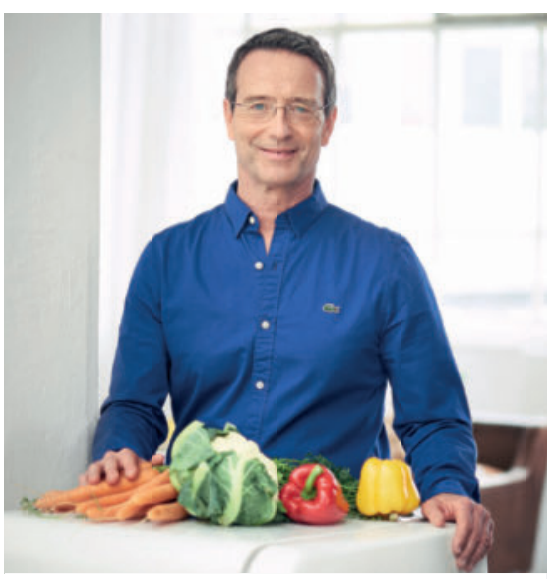

Dr. Matthias Riedl

\section{ZUR PERSON}

Dr. med. Matthias Riedl ist Internist, Diabetologe und Ernährungsmediziner. 2010 gründete er das medicum Hamburg MVZ, in dem inzwischen für über 80 Krankheiten Ernährungstherapien entwickelt wurden. Riedl engagierte sich als einer der ersten Spezialisten für eine Remissionsbehandlung von Typ-2-Diabetikern.

cker empfiehlt die WHO 25 Gramm täglich. Das sollte man anstreben. Bei 500 Gramm Gemüse ist gleichzeitig der antidiabetisch wirkende Ballaststoffanteil von 30 Gramm enthalten. Kohlenhydrate wie Kartoffeln, Reis, Nudeln und Brot brauchen v. a. Sportler, wenn sie als Vollkornvariante gewählt werden, ist auch das in Ordnung.

Das sind die Schieberegler, mit denen wir Diabetes, Übergewicht und Zivilisationskrankheiten behandeln. Die ,10 Regeln der DGE' sind zu kompliziert, wir brauchen es in der Praxis einfacher und müssen uns auf die wichtigsten Dinge konzentrieren.

\section{Das ist eigentlich recht einfach?}

Ja. Man muss lediglich die Gemüsemenge, die Eiweißmenge und die Zuckermenge zusammenzählen. Und auf das Zweibis Drei-Mahlzeiten-Prinzip achten. Das ist schon alles. 
Es wurde und wird zu schnell mit Insulin behandelt. Warum genau ist das nicht zielführend?

Die Idee war, den Patienten trotz Diabeteserkrankung eine hohe Lebensqualität zu ermöglichen. In Kombination mit Sulfonylharnstoffen ist die Insulingabe jedoch mit erhöhter Gewichtszunahme assoziiert. Manche Menschen reagieren darauf sehr empfindlich und nehmen bis zu $20 \mathrm{Ki}$ logramm pro Jahr zu. Diese Patienten sollten möglichst nicht oder so spät wie möglich mit Insulin behandelt werden.

\section{Inzwischen hat sich die Erkenntnis} durchgesetzt, dass Diabetes Typ 2 eine Lebensstilerkrankung ist, die in den meisten Fällen durch Fehlernährung, Bewegungsmangel und Stressbelastung hervorgerufen wird. Warum wird das immer noch zu wenig umgesetzt?

In meiner Generation spielt sicher noch der Einfluss der „Lebensqualität-Schule“ eine große Rolle. Es hat sich die Struktur der Diabetes-Schwerpunktpraxis herausgebildet, in der die Patienten hervorragend in der Insulintherapie geschult werden. Leider kommt in diesem Konzept die Ernährungstherapie zu kurz, bzw. sie ist zu wenig effektiv. Es gibt zwar die Medias-2-Schulung, von der manche Patienten auch profitieren, viele aber auch nicht.

\section{Die Chance einer Diabetesremission liegt bei über $80 \%$, wenn das Gewicht um 10-15 Kilo- gramm reduziert wird.}

\section{Wie gehen Sie bei sich in der Praxis vor? Was geben Sie den Patienten an die Hand?}

Erst einmal muss der Patient selbst entscheiden, was er möchte. Wir klären ihn auf, dass eine Remission von Typ-2-Diabetes möglich ist und in vielen Fällen auch, das Insulin zu reduzieren bzw. ganz davon wegzukommen. Natürlich sind die Chancen nicht bei allen Menschen gleich groß. Wer viel abnehmen kann, hat eine größere Chance als Menschen mit Normalgewicht. Die Chance einer Diabetesremission liegt bei über $80 \%$, wenn das Gewicht um 10-15 Kilogramm reduziert wird. Das schaffen die meisten Menschen aber nur mit professioneller Un- terstützung. Wir fragen die Patienten also zunächst, ob ihnen das wichtig ist und ob sie es jetzt ändern möchten.

\section{Und dann starten Sie mit der Ernährungstherapie?}

Genau. Wir beginnen mit einer Ernährungsanalyse und gehen nach dem 20:80-Prinzip vor. Die Ernährungsanalyse muss individuell erfolgen. Wir listen quasi wie bei einer Unternehmensanalyse in roten Zahlen auf, was schlecht läuft. Dann entscheidet der Patient, was er ändern möchte und in welchem Umfang. Wir versuchen, die Big Points anzugehen, je weniger, desto besser, weil zu große Veränderungen überfordern. Hinzu können Elemente wie eine ärztlich betreute Formula-Diät, Haferkuren oder auch Intervallfasten kommen. Wir arbeiten auf eine pflanzlich betonte Mischkost hin, die je nach Bewegungsgrad auch Low-Carb-Elemente enthält, aber nicht als Dogma. Wir achten auf eine ausreichende Eiweißmenge. Und schließlich setzen wir, wenn der Patient bereits mit Insulin behandelt wird, nach Möglichkeit alle dickmachenden Antidiabetika ab und ersetzen sie durch Antidiabetika, die bei der Gewichtsreduktion helfen. Das sind die Inkretin-Antidiabetika und die SGLT-2-Inhibitoren.

\section{Ist für manche Patienten nicht auch eine abrupte Ernährungsumstellung einfacher?}

Das lassen wir den Patienten entscheiden, weil es zu seinem Leben passen muss. Manche möchten sehr viel machen. Andere möchten erst einmal eine Mahlzeitenrhythmisierung versuchen, das Drei-MahlzeitenPrinzip. Viele arbeiten mit dem Drei-Mahlzeiten-Prinzip schon allein. Das ist sozusagen die Minimaleinstiegsvariante in das Intervallfasten.

Wichtig ist, am Anfang die Selbstwirksamkeit des Patienten zu aktivieren. Dazu muss er verstehen, worum es geht, dass Selbstheilung möglich ist und wie man es angehen kann. Dann setzt er es auch um.

\section{Es gibt verschiedene Messmethoden wie BMI, Bauchumfang oder Bioimpedanzanalyse. Mit welchen arbeiten Sie?}

Unser wichtigster Parameter ist die Bioimpedanzanalyse. Wir können damit einschätzen, wie die Muskelmasse und die Fettmas- se verteilt sind, wie viel Bauchfett vorhanden ist und ob eine Sarkopenie vorliegt. Diese Angaben sind vor allen Dingen auch im Therapieverlauf wichtig. Die Bioimpedanzanalyse ist sozusagen unser EKG.

\section{Ernährung ist oft unbewusst, von Gewohnheiten oder emotional} beeinflusst. Und wir sind durch unser soziales Umfeld geprägt. Welche Rolle spielen diese Prägungen?

Prägungen entstehen bereits im Mutterleib. Bekommt ein Kind im Mutterleib z. B. die ganze Bandbreite einer vegetarischen $\mathrm{Er}$ nährung mit viel Gemüse und Kräutern zu schmecken, erhöht sich die Wahrscheinlichkeit, dass es selbst ein Gemüseesser wird. In den ersten beiden Lebensjahren setzt sich die Prägung fort, nach dem zweiten Lebensjahr ist das Kind grundgeprägt. Das haben wir Menschen mit allen Primaten gemeinsam: Ein Orang-Utan-Junges z. B. lernt in den ersten beiden Lebensjahren, welche Pflanzen es verzehren kann und welche giftig sind. Das ist ein Überlebensprinzip. Dieses Prinzip funktioniert bei uns Menschen nicht mehr, weil wir in einer ernährungsfeindlichen Umgebung leben - im Supermarkt sozusagen. Man kann alles essen, und genau das ist das Problem. Hinzu kommt, dass Kinder durch das Verhalten ihrer Eltern oder Betreuer geprägt werden - zum Trost gibt es ein Bonbon oder Schokolade. Ein Kind lernt dieses Verhalten blitzschnell und wird in seinem späteren Leben das Gleiche tun. Es ist traurig, ihm ist langweilig oder es fühlt sich ungeliebt, dann greift es zur Schokolade. In meiner Generation hörten wir den Satz: Iss deinen Teller leer. Für Menschen, die zu Übergewicht neigen, kann das der Sargnagel sein.

Vor ein paar Tagen erzählte mir ein Patient mit einer Essstörung von einer Übung in der Reha: Alle sollten an einem Stück Schokolade riechen und über ihre Gefühle dabei erzählen. Er sagte: „Eigentlich habe ich gar keine Lust, die Schokolade zu essen, ich möchte einfach nur in den Arm genommen werden." Und weinte dann. Solche Beispiele zeigen immer wieder die Macht von Prägungen aus der Kindheit.

Wie gehen Sie es in der Therapie an? Wir arbeiten interdisziplinär: Ernährungsmediziner, Ernährungsberater und auch Psychologen sitzen bei uns oft gemein- 


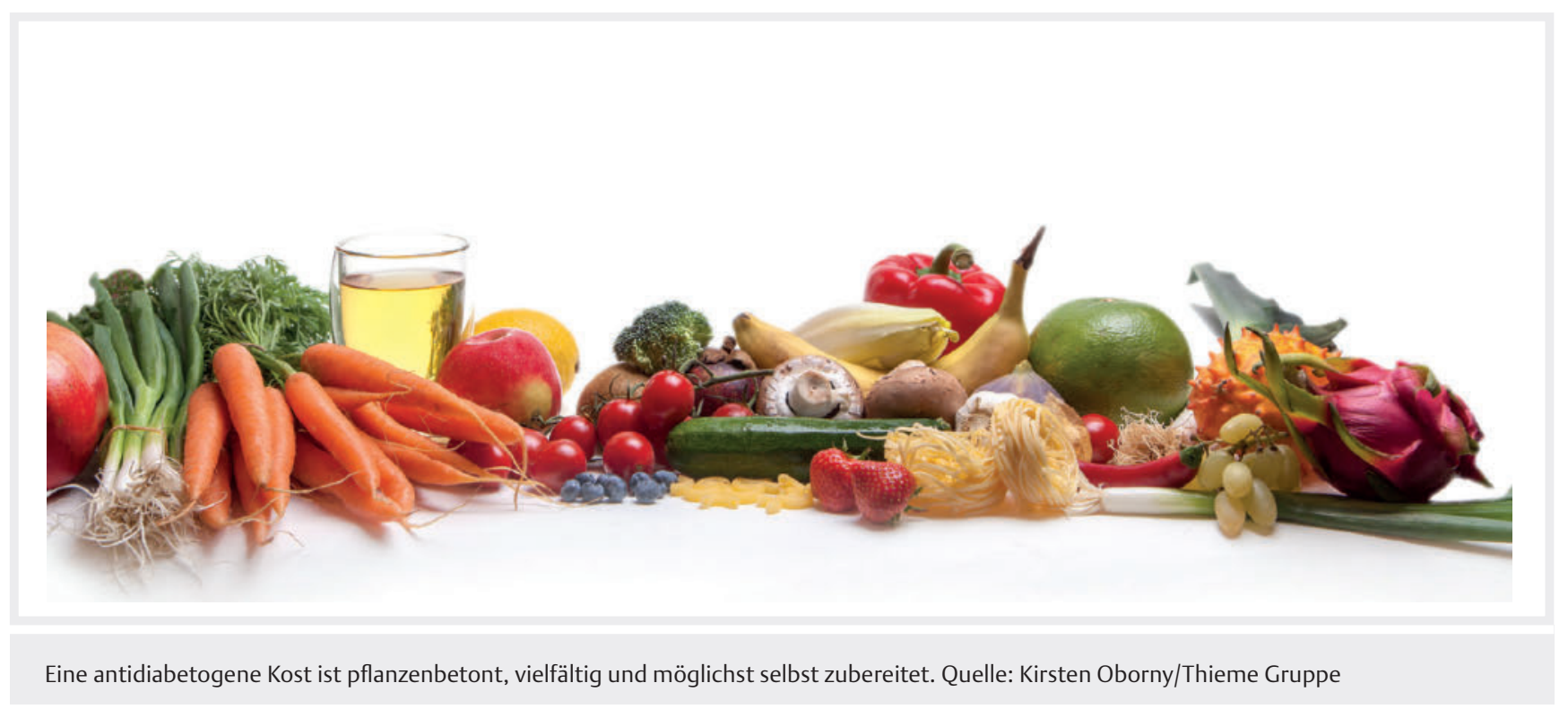

sam am Tisch. Das ist meines Erachtens eine wichtige Voraussetzung für eine ernährungsmedizinische Schwerpunktpraxis.

\section{Welche Rolle spielen bei Ihnen} Mikronährstoffe bzw. Nahrungsergänzungsmittel?

Wir versuchen den Ausgleich von Nährstoffen, die im Mangel liegen, immer zunächst durch die Ernährung zu beheben. Je nach Lebenssituation substituieren wir aber auch gezielt, wenn nötig.

\section{Das Darmmikrobiom scheint seit einigen Jahren für sehr viele Erkrankungen mitverantwortlich zu sein, u. a. auch für Adipositas. Behandeln Sie gezielt den Darm mit?} Wir führen in unserer Praxis keine aktive Darmsanierung durch, weil die wissenschaftliche Datenlage, wie und mit welchen Präparaten, noch zu dünn ist. Aber wir achten darauf, dass die Ernährung förderlich für eine gesunde Darmflora ist, $d$.h. reichlich Ballaststoffe und Gemüse, wenig Fleisch, Zucker und Alkohol. Genau das charakterisiert die artgerechte Ernährung und sie ist gleichzeitig darmgesund.

Sicher erleben Ihre Patienten trotzdem Rückschläge, fallen bei Problemen oder Stress in alte Verhaltensmuster zurück. Wie motivieren Sie sie dann wieder? Wichtig ist, mit ihnen darüber zu sprechen, was der Grund für den Rückschlag war. Und ihnen zu vermitteln, dass Rückschläge menschlich sind und niemals die Selbst- wirksamkeit beschädigen. Mit sich selbst liebenswürdig und wertschätzend umzugehen, ist ausgesprochen wichtig. Wir versuchen, die Menschen zu trösten, dass es in Ordnung ist, wenn es zu viel bei der Arbeit war oder der Hund gestorben ist. Manchmal setzen wir auch die Therapie aktiv aus und empfehlen, in einem halben Jahr weiterzumachen. Die Menschen brauchen psychische Unterstützung.

\section{Und was motiviert Patienten am meisten?}

Am meisten motiviert sie, wenn sie feststellen, wie groß die Effekte kleiner Veränderungen in der Ernährung sein können. Dann bleiben sie dabei. Und das ist der Sinn des 20:80-Prinzips. Wenn die Patienten kleine Veränderungen für ein paar Monate umgesetzt haben und sagen: Ich fühle mich fitter denn je, geistig wie körperlich. Oder bei Patienten, die eigentlich wegen ihrer Migräne zu uns kamen und neben der Symptomlinderung noch 5 Kilogramm abgenommen haben.
Bis hierhin haben wir über Diabetes Typ 2 gesprochen. Können von diesen Ernährungsempfehlungen auch Typ-1-Diabetiker profitieren?

Ja, unbedingt. Natürlich werden Typ-1-Diabetiker nie in eine Remission kommen, aber auch sie haben häufig Gewichtsprobleme. Mit einer förderlichen Ernährungsweise werden sie bessere $\mathrm{HbA}_{1 \mathrm{c}}$-Werte mit einer geringeren Schwankungsbreite haben. Auch bei Typ-1-Diabetikern rächt sich die frühere Sichtweise, den Fokus in erster Linie auf die Lebensqualität zu legen, alles essen zu können und die Insulindosis daran anzupassen.

Diabetes Typ 2 wird ja auch durch andere Lebensstilfaktoren mitverursacht. Empfehlen Sie über die Ernährung hinaus Änderungen?

Ja, unbedingt. Wenn wir uns anschauen, welche Krankheiten immer weiter zunehmen, sind es die Zivilisationskrankheiten. An den Naturvölkern sehen wir, dass das artgerechte Essen auch in ein artgerech- 
tes Leben eingebettet ist. Wir wissen, dass Waldumgebung beruhigend auf uns wirkt. Vogelgezwitscher signalisiert uns Entspannung und Sicherheit. Es geht um die Balance von Stress und Entspannung, Bewegung und Ruhe. Wir empfehlen allen Patienten, ein geeignetes Verfahren zur Stressreduktion (MBSR) zu erlernen und zu praktizieren. Dazu gehören Meditation, Stressreduktion und natürlich Sport. Auch hier ist die individuelle Beratung des Patienten entscheidend, damit er es an seine Lebenssituation anpassen und regelmäßig umsetzen kann. Neben den positiven Auswirkungen auf die Psyche wirken Sport und Stressreduktion auch der erhöhten Entzündungsbereitschaft bei einer Diabeteserkrankung entgegen.

\section{Am besten wäre sicher die Diabetes- prävention. Wie könnte die in der Praxis aussehen?}

Diabetesprävention, und nicht nur die Diabetesprävention, sollte am besten zu einem ganz frühen Zeitpunkt beginnen. Die sozialen Prägungen, zu denen auch das Essen gehört, beginnen im Mutterleib, und das hat die Gesellschaft nicht verstanden. Werdende Eltern sollten Bescheid wissen, welche Folgen eine Fehlernährung schon in der Schwangerschaft für das Kind haben kann. Und wir sollten endlich Werbung für Kindernahrungsmittel verbieten. Ab dem 3. Lebensjahr haben McDonald's \& Co. einen immensen Einfluss auf Kinder, sodass wir sie schützen sollten. Das wären meine Anregungen, wenn wir das Problem an der Wurzel packen wollen.

\section{Diabetesprävention muss zu einem ganz frühen Zeitpunkt beginnen. Das hat die Gesellschaft nicht verstanden.}

\section{Was meinen Sie, wird das Präventions- gesetz in seiner jetzigen Form dabei helfen?}

Wenn wir beim Typ-2-Diabetes bleiben, habe ich meine Zweifel. Es dreht sich im Präventionsgesetz viel um Bewegung und die ist zweifellos wichtig. Aber angesichts der immer schlechter werdenden Ernährung der Menschen in Deutschland ist sie fast zweitrangig geworden. Natürlich bil- det sie eine wichtige Basis der Prävention, aber Empfehlungen wie 10 Stunden Bewegung pro Woche gehen für viele Menschen an der Lebenswirklichkeit vorbei, weil sie es nicht schaffen.

\section{Sie sind Teil der Ernährungs-Docs, einem TV-Format, das der NDR ausstrahlt. Wie ist Ihr Eindruck, erreichen Sie damit die Menschen, für die es Relevanz hat?}

Auf jeden Fall. Ich habe den Eindruck, damit wurde eine Bewegung angestoßen, und zwar von unten. Der Online-Auftritt beim NDR ist ausgesprochen erfolgreich und wird sehr oft angeklickt. Und nicht zuletzt sehe ich in der Praxis, dass es bei den Menschen ankommt. Vor 10 Jahren noch haben wir bei den Ernährungsprotokollen der Patienten viele Ernährungsfehler gesehen. Das ist heute anders geworden. Sie haben sich bereits bevor sie zu uns kommen belesen und ihre Ernährung verändert. Bei vielen Patienten machen wir quasi nur noch Feinarbeit.

Ein extremes Beispiel ist sicher ein Patient, der 260 Kilogramm wog. Diese Menschen sind todgeweiht, wenn sie nichts ändern, da brauchen wir uns nichts vorzumachen. Er hat die Ernährungs-Docs gesehen und sich das Buch dazu gekauft („Abnehmen nach dem 20:80-Prinzip"). Darin haben wir unsere Erfahrung der letzten 20 Jahre eingebracht mit Checklisten, Rezepten und Hilfestellungen. Damit hat er in 2 Jahren 160 Kilogramm abgenommen, allein. Das ist sicher ein absoluter Ausnahmefall, aber wir sehen auch daran, dass wir so etwas verändern.

Für diese Form der Ernährungstherapie sind Gespräche enorm wichtig. Ist das in einer Kassenpraxis leistbar?

In unserem Zentrum arbeiten Experten aus verschiedenen Fachdisziplinen. Die Ernährungstherapie wird beispielsweise für Ernährungstherapeuten auf Antrag erstattet. Den Ernährungsmediziner gibt es gemäß Kassenrecht nicht, und die Behandlung wird nicht honoriert. Ich habe es 15 Jahre lang trotzdem gemacht, dann aber entschieden, dass das Defizit nicht mehr tragbar ist. Die Patienten müssen jetzt die Behandlung nach GOÄ selbst zahlen. Einfachere Fälle werden von den Ernährungstherapeuten behandelt und haben die Chance auf eine
Erstattung. Mittlerweile sagen aber viele Patienten, sie möchten die Therapie und zahlen selbst dafür.

\section{Was würden Sie sich für die Ernährungstherapie in einem idealen Gesundheitssystem wünschen?}

Die Ernährungstherapie sollte für alle Fachbereiche eine verpflichtende Option sein. Wir kennen inzwischen so viele Erkrankungen, die in einem Zusammenhang mit der Ernährung stehen. Das betrifft beispielsweise die Augenheilkunde bei der Prävention der Makuladegeneration. Oder die Zahnheilkunde bei der Behandlung der Parodontitis. In den meisten Fachrichtungen spielt die Ernährungsmedizin derzeit nahezu keine Rolle.

Dafür am ehesten offen sind nach meiner Erfahrung die Neurologie und die Orthopädie, weil die Folgen von Ernährungsfehlern wie Übergewicht und erhöhte Entzündungsbereitschaft in der Praxis besonders sichtbar werden. Ebenso könnten beispielsweise aber auch Rheumatologen oder Gynäkologen sehr davon profitieren. Fehlernährung gilt als eine der wichtigsten Ursachen für Unfruchtbarkeit. Ich denke, es ist auch eine Generationenfrage. Die jüngeren Kolleginnen und Kollegen stehen dem viel offener gegenüber.

\section{Ließe sich die große Diabetes-Welle so stoppen?}

Ja. Sie ließe sich zumindest deutlich verlangsamen, wenn wir den Menschen mehr vermitteln würden, dass die Ernährung Ursache und Therapie für ihre Erkrankung ist und wie sie es im Alltag umsetzen können.

\section{Herzlichen Dank für das Interview.}

Das Interview führte Anke Niklas. Nachdruck aus zkm - Zeitschrift für Komplementärmedizin 2-2020 mit freundlicher Genehmigung. 